\section{A Comparison between Neurofeedback and Balance Exercise on Balance of Healthy Older Adults}

\author{
Katayoon Rezaei ${ }^{1,2}$, Mohammad Nami33,4,5, Ehsan Sinaei ${ }^{6,7}$, \\ Zahra Bagheri ${ }^{8}$, Amin Kordi Yoosefinejad ${ }^{1,2 *} \odot$
}

\begin{abstract}
Background: Balance ability is a crucial component of independent daily activities among the older adultss. Balance impairment is one of the major risk factors for falls and related complications.
\end{abstract}

Objective: The present study aims to investigate and compare the effect of neurofeedback training and balance training on balance and fall risk among older adults.

Material and Methods: In this randomized controlled trial, a total of 48 older adults aged more than 65 years were recruited and randomly assigned into two groups, neurofeedback group $(n=24)$ and balance exercise group $(n=24)$. Prior to the intervention, the static balance, dynamic balance, and fall risk were measured using Biodex D balance system and Fullerton Advanced Balance scale. Subjects in neurofeedback group received neurofeedback training for 12 sessions of 30-min, every other day. Moreover, subjects in balance exercise group received balance training for four weeks in 12 sessions (45-minute) every other day. After the intervention, balance measurements were repeated in both groups. The significance level was set at $\mathrm{p}<0.05$.

Results: Static balance and dynamic balance were shown to significantly improve, after the interventions $(\mathrm{p}<0.001)$. Furthermore, fall risk was significantly reduced, after the trial $(p<0.001)$. In addition, the therapeutic effect of neurofeedback training was not less significant than exercises on balance in the older adults $(\mathrm{p}<0.001)$.

Conclusion: The findings suggest that both neurofeedback training and balance training improved balance ability among the older adults. Results also show the therapeutic effect of neurofeedback training on balance in older people. However, further research is required to accurately investigate the long-term effects of these two treatment methods among the older adults.

Citation: Rezaei K, Nami M, Sinaei E, Bagheri Z, Kordi Yoosefinejad A. A Comparison between Effects of Neurofeedback and Balance Exercise on Balance of Healthy Older Adults. J Biomed Phys Eng. 2021;11(6):713-722. doi: 10.31661/jbpe.v0i0.1203.

\section{Keywords}

Aged; Accident Falls; Physical Education and Training; Postural Balance; Neurofeedback

\section{Introduction}

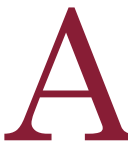
ging process is naturally accompanied by changes in musculoskeletal, vestibular, somatosensory, and visual systems [1]. Ageing also reduces physical and mental abilities resulting in the increased risk of balance impairments and fall. Balance problems lead to reducing quality of life and impair daily functioning of the older adults. Appropriate balance ability requires a complex cooperation and inte-
${ }^{1} \mathrm{PhD}$, Department of

Physical Therapy, School

of Rehabilitation Sci-

ences, Shiraz University of

Medical Sciences, Shiraz,

Iran

${ }^{2} \mathrm{PhD}$, Rehabilitation Sci-

ences Research center,

Shiraz University of Medi-

cal Sciences, Shiraz, Iran

${ }^{3} \mathrm{PhD}$, Neuroscience Labo-

ratory (Brain, Cognition

and Behavior), Depart-

ment of Neuroscience,

School of Advanced Medi-

cal Science and Technolo-

gies, Shiraz University of

Medical Sciences, Shiraz,

Iran

${ }^{4} \mathrm{PhD}$, DANA Brain Health

Institute, Iranian Neu-

roscience Society, Fars

Chapter, Shiraz, Iran

${ }^{5} \mathrm{PhD}$, Academy of Health,

Senses Cultural Founda-

tion, Sacramento, CA, USA

${ }^{6} \mathrm{MSc}$, Department of

Physical therapy, School

of Rehabilitation Sci-

ences, Shiraz University of

Medical Sciences, Shiraz,

Iran

${ }^{7} \mathrm{MSc}$, Rehabilitation Sci-

ences Research center,

Shiraz University of Medi-

cal Sciences, Shiraz, Iran

${ }^{8} \mathrm{PhD}$, Department of Bio-

statistics, Shiraz Univer-

sity of Medical Sciences,

Shiraz, Iran

*Corresponding author: Amin Kordi Yoosefinejad Department of Physical

Therapy, School of Rehabilitation Sciences, Shiraz University of Medical

Sciences, Shiraz, Iran

E-mail: yoosefinejad@

sums.ac.ir

Received: 17 June 2019 Accepted: 10 July 2019 
gration of sensory information about the surrounding environment, as well as appropriate brain function to provide desired motor function to control the body. Reduced balance and its consequences can impose a huge socioeconomic burden on the one-third and the society [2-4]. About one third of population over 65 years old have experienced at least one history of fall or impaired balance annually [3]. In addition to conventional balance exercises, there are a variety of other exercise protocols to improve balance performance in older adults, including proprioceptive neuromuscular facilitation exercises (PNF) [5, 6], single and dual task balance training [7-9], mental imagery $[10,11]$, and neurofeedback training [12].

Neurofeedback, also known as brain wave biofeedback [13], is a safe non-aggressive technique affecting many brain functions such as concentration, attention, anxiety, and learning $[14,15]$. According to the literature, neurofeedback training can effectively improve balance status in the older adults, athletes, stroke, and Parkinson patients [12, 16-18]. Neurofeedback training is applied during a few sessions in order to modify dysfunctional brain waves, and improve brain activation patterns. Indeed, this technique is used as a tool to increase or decrease specific brain waves in definite brain regions [19]. It is believed that this modern technique improves balance by modifying brain patterns in balance-controlling systems such as visual, vestibular and cerebellar systems.

A large number of studies have been carried out on the effects of exercise on balance in older adults, whereas the number of studies on modern rehabilitation techniques such as neurofeedback training is limited. Since older people have difficulties in performing physical exercises such as pain aggravation, weakness, and lack of confidence, neurofeedback and other brain training procedures might be effective alternative methods to serve the purpose of balance improvement in this population [12].
The present study was conducted to determine and compare the effects of conventional balance training and neurofeedback training on balance status of healthy older adults with mild balance impairments. If these therapeutic methods are proved to improve balance performance to the same extent, neurofeedback can be a viable alternative for the existing balance.

\section{Material and Methods}

This randomized controlled trial (ICRT 20180711040419 N1) was conducted on 48 older adults men and women ( $\geq 65$ years) with an average age of $70.35 \pm 4.57$ years in Shiraz, Iran. Randomization was performed using randomization block allocation (size of block-4). According to non-inferiority assumption, using PASS software, the sample size was determined 48 (24 in each group) with $\alpha=0.05$, power $=0.8$, margin $=0.44$ and real difference $=-1$, based on the data of a previous pertinent study [20]. Those older adults, who were able to walk independently without assistive devices, with Mini-Mental State Examination (MMSE) score below the cut-off of 24, scores lower than 7 out of 15 in geriatric depression scale (GDS), and scores between 25 and 35 on the Fullerton Advanced Balance (FAB) scale were recruited into this study $[21,22]$. Subjects with definite diagnosis of neurological or orthopedic disorders, non-corrected visual impairment, dizziness, internal ear disorders, and a history of falls more than twice during the past six months, were excluded from the study.

All participants were asked not to consume any sedative drugs prior to evaluation. This study was approved by the local medical ethics committee in accordance with the standards of Helsinki declaration. Recruitment strategy is presented in [Figure 1].

Written consent informs were assigned by all participants prior to commencement of study. Subjects were randomly assigned into two groups, including neurofeedback and bal- 


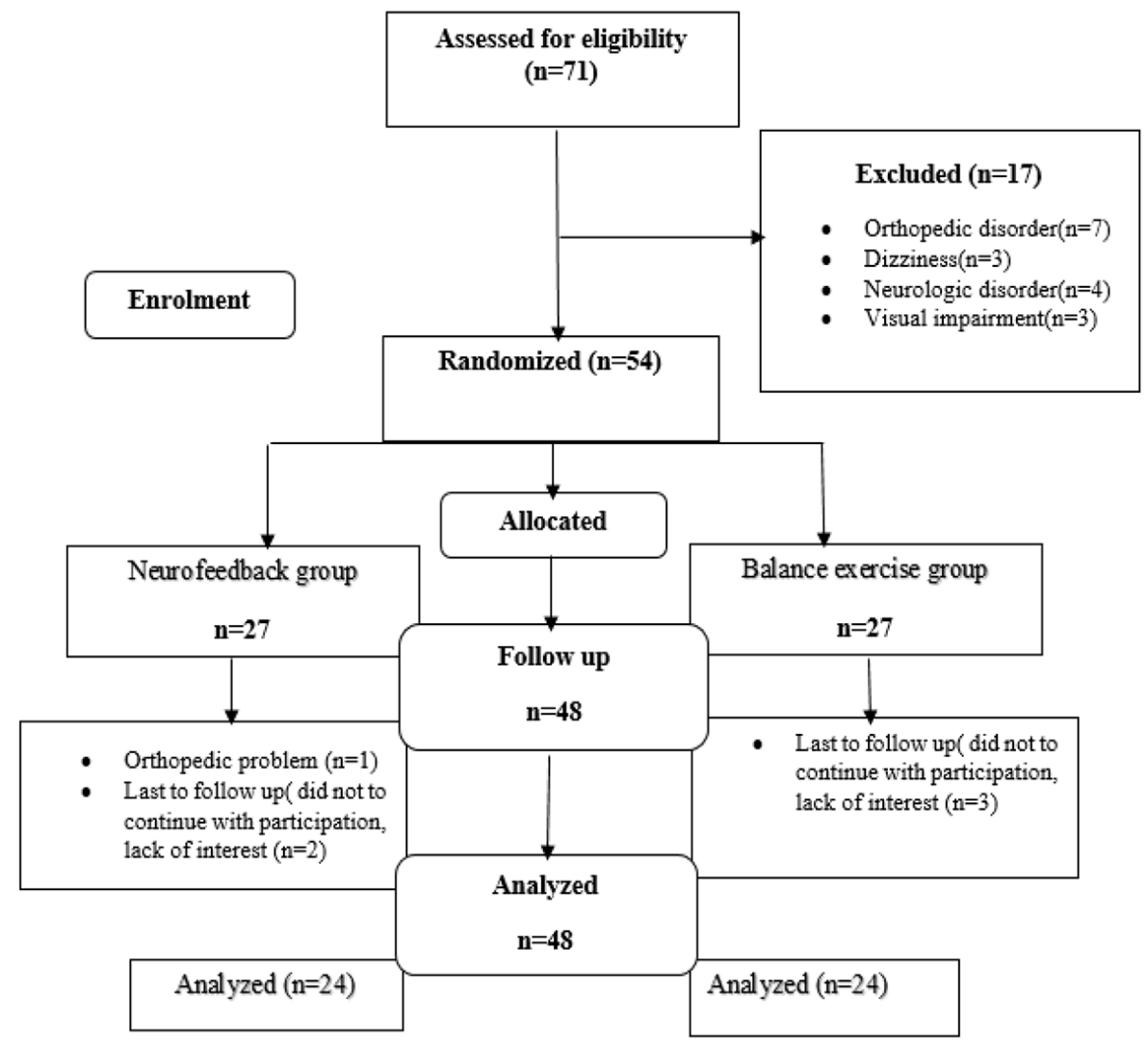

Figure 1: CONSORT flowchart

ance exercise groups. The balance level was measured by Biodex Balance system SD (Biodex Medical Systems, Inc. New York, USA) and $\mathrm{FAB}$, one day before the intervention and after the end of the intervention. All participants were evaluated by an independent assessor, who was expert in this field.

\section{Outcome measures}

The primary outcome measure was the score of fall risk on Biodex balance system. Other outcome measures such as static and dynamic stability test, as well as and FAB score, were considered as the secondary outcome measures.

In postural stability test, the subject performed three trials, each lasting 20 seconds, with 10 seconds rest between trials. This test attempts to challenge the postural-control system by measuring the angular excursion of patient's center-of-gravity on the balance board. In the other words, this testing protocol records center of gravity $(\mathrm{COG})$ and center of pressure (COP) fluctuations, and the subject was asked to maintain a fixed position during perturbation of rotating plate by holding the cursor in the middle of rotating plate [20].

In dynamic balance and fall risk tests, after entering all data related to the angles of both feet (positioning of ankle), the subject was asked to perform three trials each lasting 20 seconds, with 10 seconds rest between trials. At the onset of testing procedure, the wobble board started to rotate causing body perturbation. The subject was instructed to hold the center-of-gravity cursor in the middle of balance board. The static balance, dynamic balance, and fall risk were measured using Bio- 
dex balance device in 8 levels. Dynamic tests were adjustable from level 1 to 12 , with level 1 indicating the minimum and 12 demonstrating the maximum stability [20].

The FAB scale assesses different dimensions of balance in older adults with no severe balance problems. This scale is used as a tool to identify high-risk older adults. This scale is comprised of 10 items, each score on a 5-point scale ranged from 0 to 4 (40 in total), and score of 25 or lower indicates high risk for fall. Some advantages of this measurement tool include no ceiling effect (subjects with more repeats score higher), useful for cases with falls due to impaired sensory system, repetitive assessment of balance function during the testing procedure to evaluation of a variety of sensory systems [21]. This scale is a validated and reliable test to assess balance in functional older adults [21, 22].

\section{Interventions}

In neurofeedback group, the neurofeedback training was conducted using Neurofeedback-
SA7400A device V5.0 with Biograph Infinity software (Thought Technology Ltd, Canada). First, the subject was positioned on a comfortable chair, and the $\mathrm{O} 1$ and $\mathrm{O} 2 \mathrm{EEG}$ points were spotted on the scalp of the subject based on international 10-20 system $[12,16]$. The bipolar technique was used for electrode placement and ground electrode was placed on right ear lobe of the subjects. The nerofeedback training sessions included 12 sessions for $30 \mathrm{~min}$ every other day. Training program was based on up training of beta wave (15-18 hertz) and down training of theta wave (4-7 hertz) [11, 12]. The difficulty of training program was automatically adjusted using biograph infiniti software based on $20-80$ protocol $(80 \%$ of the beta waves were increased, and $20 \%$ of theta waves were suppressed) [Figure 2]. During the sessions, two animations (Gorilla and puzzle) were played for every $15 \mathrm{~min}$, to reduce fatigue among participants $[12,16]$.

\section{Balance exercises}

In balance exercise group, the balance train-

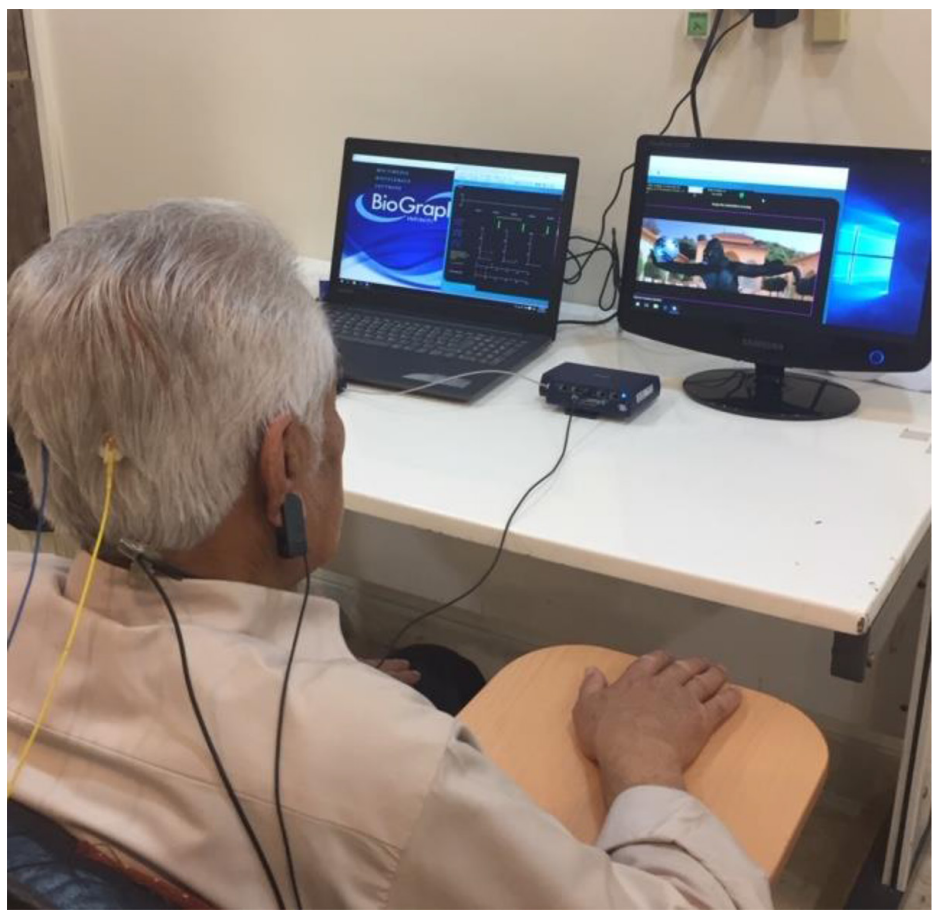

Figure 2: Neurofeedback training 
ing procedure was performed based on a protocol proposed by O'Sullivan \& Schmitz [9, 23]. Accordingly, subjects received 12 (45-minute) sessions, every other day, for four weeks as follows:

$1^{\text {st }}$ week: Normal, tandem, and single-leg standing on a firm surface, with open and then closed eyes.

$2^{\text {nd }}$ week: Semi-squat, forward and lateral lunge standing on a firm surface, and standing on foam, with open and then closed eyes.

$3^{\text {rd }}$ week: Semi-squat, tandem, and single-leg standing on foam, with open and then closed eyes.

$4^{\text {th }}$ week: Standing and semi-squat on balance board and standing on inflated disk, with open eyes.

\section{Statistical analysis}

Statistical analysis was done with SPSS software version 19 (IBM statistic, New York, NY, USA). Non- inferiority test was done by SAS (Statistical Analysis System), version 9.2. The normality of distribution of variables before intervention was evaluated by ShapiroWilk test. The values for all variables showed a normal distribution, thus parametric tests of independent $t$ - test and paired- test were used for between and within-group comparisons, respectively. In all tests, significant difference level was set as 0.05 . Effect sizes were determined as small (0.2-0.6), moderate (0.6- 1.2), and large (1.2- 2), based on the Cohen's d test [20].

\section{Results}

The present study was carried out to compare the effects of neurofeedback training and balance training on balance ability of older adults. [Figure 1] represents the flow chart of the stages of the study. According to [Table 1], the two groups were well-matched regarding the demographic characteristics, MMSE and GDS score and there was no statistically significant difference between groups at baseline [Table 1]. Based on within-group comparisons represented in [Table 2], all Biobex variables (static and dynamic balance and fall risk) decreased significantly and FAB score increased significantly following interventions, in both groups $(p<0.001)$. As reported in [Table 3], results of non-inferiority test conducted on Biodex variables after the interventions showed significant difference between the groups $(\mathrm{p}<0.001)$. This finding indicates equal effectiveness of both neurofeedback and balance exercise training

Table 1: Demographic characteristics of participants

\begin{tabular}{|c|c|c|c|}
\hline \multirow[t]{2}{*}{ Group } & $\begin{array}{l}\text { Neurofeedback group } \\
(n=24)\end{array}$ & $\begin{array}{c}\text { Balance exercise group } \\
(n=24)\end{array}$ & \multirow{2}{*}{ P-value } \\
\hline & Mean \pm SD & Mean \pm SD & \\
\hline Age (year) & $69.95 \pm 4.53$ & $70.75 \pm 4.68$ & 0.55 \\
\hline Weight (kg) & $68.70 \pm 9.90$ & $70.33 \pm 6.84$ & 0.51 \\
\hline Height (cm) & $159.70 \pm 7.64$ & $161.37 \pm 7.56$ & 0.45 \\
\hline${ }^{*} \mathrm{BMI}\left(\mathrm{kg} / \mathrm{m}^{2}\right)$ & $26.95 \pm 3054$ & $27.13 \pm 3.48$ & 0.86 \\
\hline$† \mathrm{MMSE}$ & $28.08 \pm 1.4$ & $27.63 \pm 1.6$ & 0.23 \\
\hline †GS & $1.88 \pm 1.5$ & $2.21 \pm 1.5$ & 0.39 \\
\hline
\end{tabular}

tGDS: Geriatric Depression Scale 
Table 2: Within-group comparison of outcome measures

\begin{tabular}{|c|c|c|c|c|c|c|}
\hline \multirow{3}{*}{$\begin{array}{l}\text { Group } \\
\qquad \text { Variable }\end{array}$} & \multicolumn{2}{|c|}{$\begin{array}{c}\text { Neurofeedback group } \\
(n=24)\end{array}$} & \multirow{3}{*}{ *P-value } & \multicolumn{2}{|c|}{$\begin{array}{c}\text { Balance exercise group } \\
(n=24)\end{array}$} & \multirow{3}{*}{ *P-value } \\
\hline & Pre-test & Post-test & & Pre-test & Post-test & \\
\hline & Mean \pm SD & Mean \pm SD & & Mean \pm SD & Mean \pm SD & \\
\hline Static balance & $1.25 \pm 0.92$ & $0.43 \pm 0.19$ & 0.001 & $1.80 \pm 1.15$ & $0.60 \pm 0.38$ & 0.001 \\
\hline Dynamic balance & $1.85 \pm 0.55$ & $0.86 \pm 0.26$ & 0.001 & $1.77 \pm 0.57$ & $1.11 \pm 0.42$ & 0.001 \\
\hline Fall risk & $1.77 \pm 0.66$ & $0.91 \pm 0.36$ & 0.001 & $1.86 \pm 0.78$ & $1.11 \pm 0.36$ & 0.001 \\
\hline FAB score & $30.91 \pm 3.10$ & $34.62 \pm 3.92$ & 0.001 & $30.54 \pm 2.93$ & $34.29 \pm 3.31$ & 0.001 \\
\hline
\end{tabular}

FAB: Fullerton Advanced Balance Scale

*P value was set $<0.05$

Table 3: Between-group comparisons of outcome measures after interventions

\begin{tabular}{lccc} 
Group & Neurofeedback group $(\mathbf{n = 2 4 )}$ & Balance exercise group $(\mathbf{n = 2 4 )}$ & P- value \\
\cline { 2 - 3 } Variable & Mean $\mathbf{\text { SD }}$ & Mean $\mathbf{\pm}$ SD & \\
\hline Static balance & $0.43 \pm 0.19$ & $0.60 \pm 0.38$ & $* 0.001$ \\
\hline Dynamic balance & $0.86 \pm 0.26$ & $1.11 \pm 0.42$ & $* 0.001$ \\
\hline Fall risk & $0.91 \pm 0.36$ & $1.11 \pm 0.36$ & $* 0.75$ \\
\hline FAB score & $34.62 \pm 3.92$ & $34.29 \pm 3.31$ & \\
- Non-inferiority test was used for statistical analysis & & \\
- Independent t test was used for FAB score & & \\
- FAB: Fullerton Advance balance & & \\
- * P- value was set at $<0.05$ & &
\end{tabular}

on improving balance performance on Biodex balance system. However, FAB score, which was analyzed by independent t-test, showed no significant between-group difference after interventions $(p=0.75)$ [Table 3]. As a result, balance status has improved in both groups after the interventions.

The Cohens'd test was performed, and the effect sizes of neurofeedback training and balance exercises on postural balance were reported as 1.23 and 1.4 , respectively.

\section{Discussion}

The purpose of the present study was to compare the effects of neurofeedback training and balance exercise training on static and dynamic balance and fall risk among healthy older adults. This study was carried out based on two main research questions as follows: can neurofeedback training improve balance status of older adults? "And" is the effect of neurofeedback training on balance performance was equal to that of the balance exercises?

In the present trial, static and dynamic balance and fall risk improved in the neurofeedback group, which was consistent with the previous studies having evaluated the effect of neurofeedback training in patients with bal- 
ance impairments $[12,16,24,25]$.

Similar to the previous studies, electrodes were placed at $\mathrm{O} 1$ and $\mathrm{O} 2$ points, which are in the vicinity of brain areas involved in balance control, such as cerebellum, occipital lobe, substantia nigra, and basal ganglia [12]. These points have a significant role in beta sensorimotor rhythm produced in posterior corona radiata $[12,26]$. The accurate placement of electrodes on scalp is of vital significance in enhancing balance function through proper nerve conduction [19]. Furthermore, the protocol used in this study was based on the theta suppression and beta enhancement. The cortical theta is frequently observed in children. In adolescents and young adults, however, theta wave tends to appear during meditative, drowsy, or sleeping states [27]. In contrast, beta wave is associated with conscious perception and usually appears when the person is alert during a specific physical activity [26]. It can be concluded that the amplitude of brainwaves in participants was unconsciously reconstructed to the desirable amplitude through provided feedbacks to the CNS system.

This process can help the individual to more precisely control CNS during cognitive fluctuations following aging, and subsequently improve balance ability $[16,24]$. Older adults may have learned to decrease theta, increase beta waves and maintain balance following neurofeedback training. Given the specific nature of neurofeedback training, subjects were able to regulate their brainwaves on Biodex balance system, and even maintain their balance in new testing protocols such as dynamic test or fall risk tests. These findings suggest that study participants learned to maintain their balance.

According to the results of this study, balance exercises significantly improved static and dynamic balance and reduced fall risk among older adults, which was in accordance with previous studies [3, 7, 9, 28]. Silsupadol et al, (2009) examined the effects of balance exercises on balance performance of older adults and reported improved balance under both single-task and dual-task. Previous studies evaluated balance using only functional tests, whereas the present study has applied laboratory Biodex balance test along with FAB scale.

Hernandez et al. (2008) demonstrated a linear reverse correlation between FAB score and fall risk in older adults, as one point reduction in total FAB scale score equaled to an $8 \%$ rise in the probability of falling [22]. In present study, an increase of 3 to 4 points in FAB score could reduce fall risk to about 24 to 32 percent in the participants. The present study revealed balance improvement through both laboratory and functional assessments. According to the literature, postural control is the result of cooperation and integrity of the motor, sensory and cognitive systems, all of which may undergo degenerative changes during aging [29].

Therapeutic effects of physical exercises can be attributed to improved proprioceptive input of muscles and joints such as knee, ankle, and cervical facet joints; all of which would considerably reduce following aging. A number of cognitive elements, such as attention, concentration, and planning may provide effective strategies to maintain postural control [30]. All of these elements are controlled by CNS; all of which would undergo degenerative changes, leading to impaired brain performance due to reduced number of neurons and blood supply as well as reduced free radicals in brain [9]. Physical exercises led to slow down this process through specific strategies, and subsequently improve cognitive components of balance.

According to the second assumption of this study, neurofeedback training was shown to be non-inferior to balance training at improving balance level in older adults subjects.

The findings of the present study suggested that both interventions significantly improved static and dynamic balance and reduced the fall risk in older adults. More importantly, the effect of neurofeedback training was not less 
than that of exercise balance training in improving balance ability of participants. In balance exercise group, exercises may improve balance level through boosting balance inputs from sensory systems involved in balance control. In neurofeedback training group, however, balance improvement can be attributed to the training and regulation of brainwaves. Therefore, similar to balance exercise training, neurofeedback training can be effectively used to improve balance and reduce fall risk in healthy older adults. According to results, the size effect of interventions was large in magnitude. Therefore, clinical relevancy of the findings was further confirmed.

The present study has several limitations. First, all participants of this study were healthy with no identified cognitive disorder, or postural impairments, thus the study results cannot be generalized to older adults with physical or cognitive impairments. Second, lack of a long-term follow-up was the other limitation of this study, which should be taken into consideration in future studies, in order to provide information about the durability of the therapeutic effects of neurofeedback training. Finally, emotional condition was not controlled in our study. As emotional factors could affect the attention and balance of the participants, this might have contaminated the obtained results.

\section{Conclusion}

The purpose of the present study was to compare the effects of neurofeedback training versus balance training on static and dynamic balance in healthy older adults with mild balance impairments. The findings suggest that both neurofeedback and balance exercises could improve postural balance, and neurofeedback training was as effective as balance training. Thus, both methods can be effectively applied in order to improve balance ability and consequently reduce the risk of falling in the older adults.

\section{Acknowledgment}

The present article was extracted from the $\mathrm{PhD}$ dissertation written by Katayoon Rezaei that was financially supported by Shiraz University of Medical Sciences, Shiraz, Iran (grant no. 1396-0106-16132).

\section{Authors' Contribution}

The concept of study was developed by Kordi Yoosefinejad A. and Rezaei K. The study was designed by Kordi Yoosefinejad A. and Nami M. The research work was proofread and supervised by Kordi Yoosefinejad A. The method implementation was carried out by Rezaei K. Rezaei K. and Sinaei E. Gather the data and the related literature and also help with writing of the related works. Results and Analysis was carried out by Bagheri Z. and Nami M. All the authors read, modified, and approved the final version of the manuscript.

\section{Ethical Approval}

This study was approved by the local medical ethics committee in accordance with the standards of Helsinki declaration. (Ethics code: IR.SUMS. REHAB.REC 1397.330).

\section{Funding}

The study was funded by vice chancellery of research, Shiraz University of Medical Sciences (grant number: 1396.01.06.16134).

\section{Conflict of Interest \\ None}

\section{References}

1. Gregg EW, Pereira MA, Caspersen CJ. Physical activity, falls, and fractures among older adults: a review of the epidemiologic evidence. J Am Geriatr Soc. 2000;48:883-93. doi: 10.1111/j.15325415.2000.tb06884.x. PubMed PMID: 10968291.

2. Sturnieks DL, St George R, Lord SR. Balance disorders in the elderly. Neurophysiol Clin. 2008;38:46778. doi: 10.1016/j.neucli.2008.09.001. PubMed PMID: 19026966.

3. Paquette MR, Li Y, Hoekstra J, Bravo J. An 8-week reactive balance training program in older healthy adults: A preliminary investigation. Journal of Sport and Health Science. 2015;4:263-9. doi: 10.1016/j.jshs.2014.06.004.

4. Aartolahti E, Tolppanen AM, Lonnroos E, Har- 
The Effect of Neurofeedback on Balance in Older Adults

tikainen S, Hakkinen A. Health condition and physical function as predictors of adherence in long-term strength and balance training among community-dwelling older adults. Arch Gerontol Geriatr. 2015;61:452-7. doi: 10.1016/j.archger.2015.06.016. PubMed PMID: 26183202.

5. Pereira MP, Gonçalves M. Proprioceptive neuromuscular facilitation improves balance and knee extensors strength of older fallers. ISRN Rehabilitation. 2012;2012. doi: 10.5402/2012/402612.

6. Young KJ, Je CW, Hwa ST. Effect of proprioceptive neuromuscular facilitation integration pattern and swiss ball training on pain and balance in elderly patients with chronic back pain. J Phys Ther Sci. 2015;27:3237-40. doi: 10.1589/jpts.27.3237. PubMed PMID: 26644682. PubMed PMCID: PMCPMC4668173.

7. Nematollahi A, Kamali F, Ghanbari A, Etminan Z, Sobhani S. Improving Balance in Older People: A Double-Blind Randomized Clinical Trial of Three Modes of Balance Training. J Aging Phys Act. 2016;24:189-95. doi: 10.1123/japa.2014-0286. PubMed PMID: 26215224.

8. Khan K, Ghous M, Malik AN, Amjad MI, Tariq I. Effects of turning and cognitive training in fall prevention with dual task training in elderly with balance impairment. Rawal Medical Journal. 2018;43:124-8.

9. Sinaei E, Kamali F, Nematollahi A, Etminan Z. Comparing the effects of balance training with and without cognitive tasks on the quality of life and balance performance in community-dwelling older adults: a single-blind randomized clinical trial. Journal of Rehabilitation Sciences \& Research. 2017;3:91-6.

10. Aslankhani MA, Shams A, Shamsipour Dehkordi $P$. Mental, physical and mixed practice effects on elderly static and dynamic balance. Iranian Journal of Ageing. 2008;3:22-9.

11. Moshref-Razavi S, Sohrabi M, Sotoodeh MS. Effect of Neurofeedback Interactions and Mental Imagery on the Elderly's Balance. Iranian Journal of Ageing. 2017;12:288-99. doi: 10.21859/sija.12.3.288.

12. Azarpaikan A, Torbati HT, Sohrabi M. Neurofeedback and physical balance in Parkinson's patients. Gait Posture. 2014;40:177-81. doi: 10.1016/j.gaitpost.2014.03.179. PubMed PMID: 24746035.

13. Hammond DC. What is neurofeedback? J Neurother. 2007;10:25-36. doi: 10.1300/j184v10n04_04.

14. Nan W, Qu X, Yang L, Wan F, Hu Y, Mou P, et al, editors. Beta/theta neurofeedback training effects in physical balance of healthy people. World Congress on Medical Physics and Biomedical En- gineering; Toronto, Canada: Springer; 2015.

15. Hammond DC. What is neurofeedback: An update. I Neurother. 2011;15:305-36. doi: 10.1080/10874208.2011.623090.

16. Hammond DC. Neurofeedback to improve physical balance, incontinence, and swallowing. J Neurother. 2005;9:27-36. doi: 10.1300/j184v09n01_03.

17. Rezaye S, Eskandarnejad M, Mohammadzadeh $\mathrm{H}$, Abedini M. Effect of neurofeedback training on dual balance tasks of motor and cognitive in older men. J Rehab Med. 2015;4:18-26.

18. Paul M, Ganesan S, Sandhu JS, Simon JV. Effect of Sensory Motor Rhythm Neurofeedback on Psycho-physiological, Electro-encephalographic Measures and Performance of Archery Players. Ibnosina Journal of Medicine \& Biomedical Sciences. 2012;4.

19. Hammond DC. Neurofeedback for the enhancement of athletic performance and physical balance. The Journal of the American Board of Sport Psychology. 2007;1:1-9.

20. Gusi N, Adsuar JC, Corzo H, del Pozo-Cruz B, Olivares PR, Parraca JA. Balance training reduces fear of falling and improves dynamic balance and isometric strength in institutionalised older people: a randomised trial. J Physiother. 2012;58:97-104. doi: 10.1016/s1836-9553(12)70089-9.

21. Rose DJ, Lucchese N, Wiersma LD. Development of a multidimensional balance scale for use with functionally independent older adults. Arch Phys Med Rehabil. 2006;87:1478-85. doi: 10.1016/j. apmr.2006.07.263. PubMed PMID: 17084123.

22. Hernandez D, Rose DJ. Predicting which older adults will or will not fall using the Fullerton Advanced Balance scale. Arch Phys Med Rehabil. 2008;89:2309-15. doi: 10.1016/j. apmr.2008.05.020. PubMed PMID: 18976981.

23. Monjezi S, Negahban H, Tajali S, Yadollahpour N, Majdinasab N. Effects of dual-task balance training on postural performance in patients with Multiple Sclerosis: a double-blind, randomized controlled pilot trial. Clin Rehabil. 2017;31:234-41. doi: 10.1177/0269215516639735. PubMed PMID: 27006419.

24. Shaw L, Zaichkowsky L, Wilson V. Setting the balance: Using biofeedback and neurofeedback with gymnasts. J Clin Sport Psychol. 2012;6:47-66. doi: 10.1123/jcsp.6.1.47.

25. Azarpaikan A, Taheri Torbati H. Effect of somatosensory and neurofeedback training on balance in older healthy adults: a preliminary investigation. Aging Clin Exp Res. 2018;30:745-53. doi: 10.1007/s40520-017-0835-3. PubMed PMID: 
29063490.

26. Halder S, Varkuti B, Bogdan M, Kubler A, Rosenstiel W, Sitaram $R$, et al. Prediction of braincomputer interface aptitude from individual brain structure. Front Hum Neurosci. 2013;7:105. doi: 10.3389/fnhum.2013.00105. PubMed PMID: 23565083. PubMed PMCID: PMCPMC3613602.

27. Dickson CT, Kirk IJ, Oddie SD, Bland BH. Classification of theta-related cells in the entorhinal cortex: cell discharges are controlled by the ascending brainstem synchronizing pathway in parallel with hippocampal theta-related cells. Hippocampus. 1995;5:306-19. doi: 10.1002/hipo.450050404. PubMed PMID: 8589794.

28. Hamed A, Bohm S, Mersmann F, Arampatzis A. Exercises of dynamic stability under unstable conditions increase muscle strength and balance ability in the elderly. Scand J Med Sci Sports. 2018;28:961-71. doi: 10.1111/sms.13019. PubMed PMID: 29154407.

29. Park JH. The effects of eyeball exercise on balance ability and falls efficacy of the elderly who have experienced a fall: A single-blind, randomized controlled trial. Arch Gerontol Geriatr. 2017;68:1815. doi: 10.1016/j.archger.2016.10.006. PubMed PMID: 27835770.

30. Bakos DS, Couto MCPDP, Melo WV, Parente MADM, Koller SH, Bizarro L. Executive functions in the young elderly and oldest old: a preliminary comparison emphasizing decision making. Psychol Neurosci. 2008;1:183-9. doi: 10.3922/j. psns.2008.2.011. 